professors with fourteen assistant lecturers, and the students in all departments were 556 in 1916. The fees for instruction are 30 francs for each semester, together with certain special fees, and foreigners are charged treble fees. The six schools have a total of 2427 students, and are fully equipped with laboratories for experimental instruction. On leaving these schools the students enter the Union of Swiss Technicians, which association now counts its members by thousands and has for its organ the Swiss Technical Review, which publishes much good original work. Altogether these institutions have proved a great success and have been of material benefit in training a large body of men for the industries, many of whom have afterwards qualified for high industrial or administrative positions at home or abroad.

\section{GRAVITATION AND THE PRINCIPLE OF RELATIVITY.1}

\section{II}

$\mathrm{W}$ $\mathrm{E}$ have to admit, then, that a world-line can be bent by the proximity of other world-lines. It can also be bent, as you see, by the proximity of my thumb. The suggestion arises, May not the two modes of bending be essentially the same? The bending by my thumb (a mathematical transformation of space and time) is in a sense spurious; the world-line is pursuing a course which is straight relative to the original material. Or we may perhaps best put it this way-the world-line still continues to take the shortest path between two points, only it reckons distance according to the length that would be occupied in the unstretched state of the bladder. It is suggested that the deflection of a world-line by gravitation is of the same nature; from each world-line a state of distortion radiates, as if from a badly puckered seam, and any other world-line takes the shortest course through this distorted region, which would immediately become straight if the strain could be undone. The same rule - of shortest distance as measured in the undistorted state-is to hold in all cases. This is a mode of reasoning which has often been fruitful in scientific generalisations. A magnetic needle turns towards the end of a bar-magnet; it also turns towards a spot near the pole of the earth; hence the suggestion that the earth is a magnet. We assume the essential identity of the two modes of deflecting the needle. It is a daring step to apply the analogy and assume the essential identity of the two ways of deflecting world-lines; but at any rate we shall make this assumption and see what comes of it.

You will see that according to this view the earth moves in a curved orbit, not because the sun exerts any direct pull, but because the earth is trying to find the shortest way through a space and time which have been tangled up by an influence radiating from the sun. We can continue to describe this indirect influence of the sun on the earth's motion as a "force"; but, assuming that it makes itself felt as a modification or strain of space and time, we are able to bring the discussion of the laws of this force into line with the discussion of the laws of space and time, i.e. the laws of geometry. Needless to say, we could not determine a physical law like the law of gravitation by geometrical reasoning without making some assumption.

I am afraid that to talk of a force as being a distortion of space and time must at first appear to you hopeless jargon. But it must be remembered first that we are not concerned with any metaphysical space and time. We mean by space and time simply a scaffold-

1 Discourse delivered at the Royal Institution on Friday, February $\mathrm{r}$, by NO. 2524, VOL. IOI] ing that we construct as the result of our measures; and if anything queer happens to our measuring apparatus, the scaftolding may easily go crooked. Taking our everyday conception of space, we should say that this room is at rest; we have been told that it is being carried round the earth once a day, but in practical life we never pay any attention to that. The space that we naturally use is thus different from, and it is not difficult to show that it is distorted as compared with, the more fundamental astronomical space in which this room is travelling at a great velocity. So our scaffolding is crooked. But, it may be asked, in what way can this distortion of our space-scaffolding be regarded as a force? The answer is quite simple. We perceive it as a force, and that is the only way in which we do perceive it. We do not perceive that this room is being carried round by the earth's rotation, but we perceive a certain force-the earth's centrifugal force. It is rather difficult to demonstrate this force, because gravitation predominates overwhelmingly; but if gravity were annihilated we should have to be tied down to the floor to prevent our flying up to the ceiling, and we should certainly feel ourselves pulled by a very vigorous centrifugal force. That is our only perception of the crookedness of our scaffolding.

We often call the centrifugal force an "unreal" force, meaning that it arises simply from a transformation of the framework of reference. Can we feel confident that gravitation is in any sense more "real"? In effect they are so much alike that even in scientific work we speak of them in one breath. What is called the value of gravity in London, $98 \mathrm{I} \cdot \mathrm{I} 7 \mathrm{~cm} . / \mathrm{sec}^{2}$, is really made up partly of the true attraction of the earth and partly of the centrifugal force. It is not considered worth while to make any distinction. Surely, then, it is not a great stretch of the imagination to regard gravitation as of the same nature as centrifugal force, being merely our perception of the crookedness of the scaffolding that we have chosen.

If gravity and centrifugal force are manifestations of the same underlying condition, it must be possible to reduce them to the same laws; but we must express the laws in a manner which will render them comparable. There is a convenient form of Newton's law, which was given by Laplace and is well known to mathematicians, which describes how the intensity at any point is related to the intensity at surrounding points-or, according to our interpretation, how the distortion of space at any point fits on to the distortion at surrounding points. It is evidently an attempt to express the general laws of the strains in space and time which occur in Nature. If we are correct in our assumption that gravitation involves nothing more than strain of space-time, ${ }^{2}$ so that its law expresses merely the relation between adjacent strains which holds by some natural necessity, clearly the strains which give the centrifugal force must obey the same general law. Here a very interesting point arises. We cannot reconcile the Newtonian law of gravitation with this condition. Newton's law and the law of centrifugal force are contradictory.

To put the matter another way, if we determine the strains by Newton's law, we get results closely agreeing with observation, provided Minkowski's space-time is used; but if we avail ourselves of our right to use a transformed space-time, the results no longer agree with observation. 'That means that Newton's law in. volves something which is not fully represented by strains, and so does not agree with our assumption. We must abandon either our assumption, or the famous law which has been accepted for more than

2 The irea is that matter represents a seam or nucleus of strain, and the strains at other points link themse'ves on arcord ng to laws inherent in the continum and quite independent of the matter. The matter sta'ts the strain but does not controi it as it goes outwards. 
200 years, and find a new law of gravitation which will fall in with our requirements.

This amended law has been found by Einstein. It appears to be the only possible law that meets our requirements, and in the limited applications which come under practical observation is sufficiently close to the old law that has served so well. In practical applications the two laws are indistinguishable, except for one or two crucial phenomena to which reference will be made later. But in gravitational fields far stronger than any of which we have experience, and for bodies moving with velocities much greater than those of the planets, the difference-would be considerable.

This idea of the distortion of space as the modus operandi of gravitation has led to a practical resulta new law of gravitation. It is not brought in as a hypothetical explanation of gravitation; if Einstein's theory is true, it is simply of the nature of an experimental fact.

If we draw a circle on a sheet of paper and measure the ratio of the circumference to the diameter, the result gives, if the experiment is performed accurately enough, the well-known number $\pi$, which has been calculated to 707 places of decimals. Now place a heavy particle at or near the centre and repeat the experiment; the ratio will be not exactly equal to $\pi$, but a little less. The experiment has not been performed, and is not likely to be performed, because the difference to be looked for is so small; - but, if Einstein's theory is correct, that must be the result. The space around the heavy particle does not obey ordinary geometry; it is non-Euclidean. The change in its properties is not metaphysical, but something which, with sufficient care, could be measured. You can keep to. Euclidean space if you like, and say that the measuring-rod has contracted or expanded according as it is placed radially or transversely to the gravitational force. That is all very well if the effect is small, but in a very intense gravitational field it would lead to ridiculous results like those we noticed in connection with the Michelson-Morley experiment-everything expanding or contracting as it changed position, and no one aware of any change going on. I think we have learnt our lesson that it is better to be content with the space of experience, whether it turns out to be Euclidean or not, and to leave to the mathematician the transformation of the phenomena into a space with more ideal properties.

This consequence of the new law of gravitation, though theoretically observable, is not likely to be put to any practical test either now or in the immediate future. But there are other consequences which just come within the range of refined observation, and so give an immediate practical importance to the new theory, which has indeed scored one very striking success. If we could isolate the sun and a single planet, then under the Newtonian law of gravitation the planet would revolve in an ellipse, repeating the same orbit indefinitely. Under the new law this is not quite true; the orbit is nearly an ellipse, but it does not exactly close up, and in the next revolution the planet describes a new ellipse in a slightly advanced position. In other words, the elliptic orbit slowly turns round in the same direction in which the planet is moving, so that after the lapse of many centuries the orbit will point in a different direction. The rate at which the orbit turns depends on the speed of motion of the planet in its orbit, so we naturally turn to the fastest moving planets, Mercury, Venus, and the earth, to see if the effect can be detected. Mercury moves at thirty miles a second, Venus at twenty-two, the earth at eighteen and a half. But there is a difficulty about Venus and the earth. Their orbits are nearly circular, and you cannot tell in which direction a circle is pointing.
Mercury combines the favourable conditions of a high speed and a satisfactorily elongated orbit the direction of which at any time can be measured with considerable precision. It is found by observation that the orbit of Mercury is advancing at the rate of 574 seconds of arc a century. This is in great measure due to the attraction of the other planets, which are pulling the orbit out of shape and changing its position. The amount of this influence can be calculated very accurately, and amounts to 532 seconds per century. There is thus a difference of forty-two seconds a century unaccounted for; and this has for long been known as one of the most celebrated discordances between observation and gravitational theory in astronomy. It is thirty times greater than the probable error which we should expect from uncertainties in the observations and theory. There are other puzzling discordances, especially in connection with the motion of the moon; but the conditions in that case are more complicated, and $I$ scarcely think they offer so direct a challenge to gravitational theory. Now Einstein's theory predicts that there will be a rotation of the orbit of Mercury additional to that produced by the action of the planets; and it predicts the exact amount-namely, that in one revolution of the planet the orbit will advance by a fraction of a revolution equal to three times the square of the ratio of the velocity of the planet to the velocity of light. We can work that out, and we find that the advance should be forty-three seconds a century-just about the amount required. Thus, whilst the Newtonian law leaves a discordance of more than forty seconds, Einstein's law agrees with observation to within a second or so.

Of course this superiority would be discounted if we could find some other application where the old Newtonian law had proved the better. But that has not happened. In all other cases the two laws agree so nearly that it has not been possible to discriminate between them by observation. The new law corrects the old where the old failed, and refrains from spoiling any agreement that already exists. The next best chance of applying the new theory is in the advance of the orbit of Mars; here Einstein's new law "gilds refined gold" by slightly improving an agreement which was already sufficiently good-a "wasteful and ridiculous excess," which is at any rate not unfavourable to the new theory.

There is another possibility of testing Einstein's theory, which it is hoped to carry out at the first opportunity. This relates to the action of gravitation on a ray of light. It is now known that electromagnetic energy possesses, the property of inertia or mass, and probably the whole of the mass of ordinary matter is due to the electromagnetic energy which it contains. Light is a form of electromagnetic energy, and there fore must have mass-a conclusion which has been found true experimentally, because light falling on any object exerts a pressure just as a jet of water would We ordinarily measure mass in pounds, and it is quite proper to speak of " a pound of light," just as we speak of a pound of tobacco. In case anyone should be thinking of going to an electric light company to buy a pound of light, I had better warn you that it is a rather expensive commodity. They usually prefer to sell it by a mysterious measure of their own, called the Board of Trade unit, and charge at least $3 d$. a unit. At that rate I calculate that they would let you have a pound of light for $\mathrm{I}_{4} \mathrm{r}, 6 \mathrm{r}_{5}, 000 \mathrm{l}$. Fortunately, we get most of our light free of charge, and the sun showers down on the earth 160 tons daily. It is just as well we are not asked to pay for it.

But although light has mass, it does not follow that light has weight. Ordinarily, mass and weight are associated in a constant proportion, but whether this

NO. 2524 , VOL. IOI $]$ 
is so in the case of light can be settled only by experiment-by weighing light. It seems that it should be just possible to do this. If a beam of light passes an object which exerts a gravitational attraction, then, if it really has weight, it must drop a little towards the object. Its path will be bent just as the trajectory of a rifle bullet is curved owing to the weight of the bullet. The velocity of light is so great that there is only one body in the solar system powerful enough to make an appreciable bend in its path, namely, the sun. If we could see a star close up to the edge of the sun, a ray of light coming from the star would bend under its own weight, and the star would be seen slightly displaced from its true position. During a total eclipse stars have occasionally been photographed fairly close to the sun, and with care it should be possible to observe this effect. There is a magnificent opportunity next year when a total eclipse of the sun takes place right in the midst of a field of bright stars. This is the best opportunity for some generations, and it is hoped to send out expeditions to the line of totality to weigh light according to this method.

In any case, great interest must attach to an attempt to settle whether or not light has weight. But there is an additional importance, because it can be made a means of confirming or disproving Einstein's theory. On Einstein's theory light must certainly have weight, because mass and weight are viewed by it as two aspects of the same thing; but his theory predicts a deflection twice as great as we should otherwise expect. Apart from surprises, there seem to be three possible results:- (I) A deflection amounting to $\mathrm{r} \cdot 75^{\prime \prime}$ at the limb of the sun, which would confirm Einstein's theory; (2) a deflection of $0.83^{\prime \prime}$ at the limb of the sun, which would overthrow Einstein's theory, but establish that light was subject to gravity; (3) no deflection, which would show that light, though possessing mass, has no weight, and hence that Newton's law of proportionality between mass and gravitation has broken down in another unexpected direction.

The purpose of Einstein's new theory has often been misunderstood, and it has been criticised as an attempt to explain gravitation. The theory does not offer any explanation of gravitation; that lies quite outside its scope, and it does not even hint at a possible mechanism. It is true that we have introduced a definite hypothesis as to the relation between gravitation and a distortion of space; but if that explains anything, it explains not gravitation, but space, i.e. the scaffolding constructed from our measures. Perhaps the position reached may be made clearer by another analogy. Let us picture the particle which describés a world-line as hurdleracer in a field thickly strewn with hurdles. The particle in passing from point to point always takes the path of least effort, crossing the fewest possible hurdles ; if the hurdles are uniformly distributed, corresponding with undistorted Minkowskian space, this will, of course, be a straight line. If the field is now distorted by a mathematical transformation such as an earthquake so that the hurdles become packed in some parts and spread out in others, the path of least effort will no longer be a straight line; but it is not difficult to see that it passes over precisely the same hurdles as before, only in their new positions. The gravitational field due to a particle corresponds with a more fundamental rearrangement of the hurdles, as though someone had taken them up and replanted them according to a law which expresses the law of gravitation. Any other particle passing through this part of the field follows the guiding rule of least effort, and curves its path, if necessary, so as to jump the fewest hurdles. Now, we have usually been under the impression that when we measured distances by physical experiments we were surveying the field, and the results could be plotted on a map; but it is now realised that we cannot do that The field itself has nothing to do with our measurements; all we do is to count hurdles. If the only cause of irregularity of the hurdles were earthquakes (mathematical transformations), that would not make much difference, because we could still plot our counts of hurdles consistently as distances on a map; and the map would represent the original condition of the field with the hurdles uniformly spaced. But the more farreaching rearrangement of hurdles by the gravitational field forces us to recognise that we are dealing with counts of hurdles and not with distances; because if we plot our measures on a map-they will not close up. The number of hurdles in the circumference of a circle ${ }^{3}$ will not be $\pi$ times the number in the diameter; and when we try to draw on a map a circle the circumference of which is less than $\pi$ times its diameter, we get into difficulties-at least in Euclidean space. This analogy brings out the point that the theory is an explanation of the real nature of our measures rather than of gravitation. We offer no explanation why the particle always takes the path of least effort-perhaps, if we may judge by our own feelings, that is so natural as to require no explanation. More seriously, we know that in consequence of the undulatory theory of light, a ray traversing a heterogeneous medium always takes the path of least time; and one can scarcely resist a vague impression that the course of a material particle may be the ray of an undulation in five dimensions. What concerns gravitation more especially is that we have offered no explanation of the linkages by which the hurdles rearrange themselves on a definite plan when disturbed by the presence of a gravitating particle; that is a point on which a mechanical theory of gravitation ought to throw light.

From the constant of gravitation, together with the other fundamental constants of Nature-the velocity of light and the quantum of action-it is possible to form a new fundamental unit of length. This unit is $7 \times 10^{-28} \mathrm{~cm}$. It seems to be inevitable that this length must play some fundamental part in any complete interpretation of gravitation. (For example, in Osborne Reynolds's theory of matter this length appears as the mean free-path of the granules of his medium.) In recent years great progress has been made in knowledge of the excessively minute; but until we can appreciate details of structure down to the quadrillionth or quintillionth of a centimetre, the most sublime of all the forces of Nature remains outside the purview of the theories of physics.

\section{UNIVERSITY AND EDUCATIONAL INTELLIGENCE.}

Cambridge. - The syndicate appointed to consider the Previous examination has issued its report. The recommendations involve changes which, if passed by the Senate, will greatly alter the present character of the examination. The syndicate advises the discontinuance of Greek as a compulsory subject, and recommends that every candidate shall be required to take at least one paper in natural science. It proposes that the examination should be in three parts: (I) Languages; (2) mathematics and natural science; (3) English subjects. In part (I) it is recommended that Latin should continue to be a compulsory subject, and that a candidate should be required to take one other foreign language, namely, Greek, French, German, or Spanish.

3 A circle would naturally be defined as a curve such that the number of hurdles (counted along the path of least effort) between any point on it and a fixed point called the centre is constant. To make the vague analogy an definite, we may suppose that the hurdles are pivoted, and awing mor automatically to face the jumper introduce into his the jumper; he is not a lowed to dodge them, i.e. to hurdles 